\title{
Post Chemo Lung Toxicity: A Less Visited Domain
}

\author{
Sidrah Saeed', Abdul Majid', Zeenat Adil', Mahnoor Rehman', Anisa Sandal', Sana lqbal' ${ }^{2}$
}

\begin{abstract}
Background: Post-anticancer chemotherapy changes in lung parenchyma is an important and challenging aetiology for radiologists presenting not uncommonly in cancer patients. Early diagnosis of post-chemotherapy lung toxicity have serious implications on patient's health.

Objective: To evaluate post-chemotherapy cancer patients for pulmonary toxicity and establish their association with frequency of chemotherapy cycles and time lapse since last cycle.

Material and Methods: This retrospective study was conducted in Kuwait teaching Hospital from January 2018 till December 2018. A total of 204 patients were evaluated with age ranging from 3 to 78 years including 63 male and 141 female patients. Chest CT scan had been done on16 slice Toshiba CT scanner in Radiology department of Kuwait Teaching Hospital. Images were evaluated in axial, coronal and sagittal planes and in lung and soft tissue window on workstation. Data like patient age ,gender , malignancy, number of chemotherapy cycles received, time lapse since last chemotherapy cycle, common CT manifestations of lung toxicity and CT lung findings unrelated to chemotherapy regimens; was collected and subjected to statistical analysis.

Results: Out of the total 204 patients, who already had anticancer therapy, 135 patients (66.2\%) did not show any pulmonary abnormality on CT chest . 11 patients (5.4\%) showed chemotherapy related pulmonary findings on CT. 46 patients (22.5\%) showed CT chest findings which were incidental and irrelevant to chemotherapy.12 patients $(5.9 \%)$ had both chemotherapy related pulmonary toxicity changes and incidental findings. The types of post chemotherapy CT lung changes were divided into groundglass haze, ground glass haze with bronchiectasis, ground glass haze with septal thickening giving crazy paving appearance, patches of consolidation and reticular thickening. 10 patients had sole finding of ground glass haze only (43.5\%) whereas 4 patient showed ground glass haze with bronchiectatic changes $(17.4 \%)$ and 2 patients depicted a crazy paving appearance $(8.7 \%)$. Significant association $(p=0.00)$ was noted between number of chemotherapy cycles and above mentioned CT lung findings .No positive correlation was present between time lapse since last chemotherapy cycle and these CT lung findings.

Conclusion: Ground glass attenuation was found to be the most profounding feature of post chemotherapy lung CT changes.

Key Words: Post Chemotherapy, Lung toxicity, Ground Glass haze, Cryptogenic Organizing pneumonia, obliterative bronchiolitis, pulmonary haemorrhages.
\end{abstract}

This article may be cited as: Saeed S, Majid A, Adil Z, Rehman M, Sandal A, Iqbal S.

Post Chemo Lung Toxicity: A Less Visited Domain. J Saidu Med Coll Swat 2020;10(2): 140-146

\section{INTRODUCTION}

Chemotherapy is the mainstay of cancer treatment once it is in advanced stages .However owing to cytotoxic effects, they are known to have delirious effects on unwanted targets ${ }^{1,2}$.

Many international studies have been conducted concentrating upon pulmonary toxicity due to chemotherapeutic drugs and their relation with development of interstitial lung diseases claiming there incidence to be as high as $10 \%^{3-7}$. Time interval between start of chemotherapy and appearance of lung changes can vary greatly from as little as 4 weeks to more than 10 weeks ${ }^{8}$.

Several studies have associated dose of chemotherapeutic agents with risk of developing pulmonary toxicity.

\section{Kwait Teaching Hospital, Peshawar. \\ 2.North West General Hospital, Peshawar}

Correspondence: Dr. Sidrah Saeed

Senior Registrar Kuwait teaching Hospital, Peshawar

Email:drsidrahsaeed233@yahoo.com
Radiological picture of chemotherapy related pulmonary toxicity is usually vague. Most commonly associated radiological findings on chest CT are ground glass haze (with or without septal thickening), patches of consolidations and bronchiectatic changes. ${ }^{3,4}$ Furthermore symptoms related to chemotherapy induced lung diseases are non-specific as well and do not correlate with radiological picture making the picture even more confusing. Frequently associated symptoms include shortness of breath, cough, chest pain however the patient might be as well be asymptomatic. ' Hence, high level of radiological suspicion is necessary for early recognition so as to avoid fatal out comes. Withdrawal of offending drug and associated steroid therapy is usually curative in early stages making early diagnosis on the part of radiologist even more necessary. ${ }^{10}$

Unfortunately chemotherapy related pulmonary changes on CT chest are usually under interpreted or ignored altogether specifically in follow up CT scans, delaying timely diagnosis. That is exactly the reason why the data in our region in this regard is scanty with only few studies inducted in Pakistan. ${ }^{11}$ 
Advent of newer chemotherapy agents has added to difficulty in interpretation of post chemotherapy related CT pulmonary change. Novel chemotherapy agents interfere with DNA and RNA synthesis and there adverse effects are well studied .12, ${ }^{13,}{ }^{14}$.In newer agents molecular therapies target specifically cancer cell surface antigen (monoclonal antibodies) and various signalling molecules (like kinase inhibitors) effecting unwanted target cells and resulting in pulmonary toxicity. ${ }^{15}$ Studies regarding these agents are underway and need further cautiousness on the part of radiologist.

Multi detector CT remains a valuable tool for investigation and evaluation of these pulmonary changes, the reason being no well-defined set of clinical presentation, history or pathological findings which had been agreed upon.

Different pathological processes are involved in pulmonary damage resulting in different radiological manifestations including diffuse alveolar damage, nonspecific interstitial pneumonia, cryptogenic organising pneumonia, obliterative bronchiolitis, pulmonary haemorrhages, edema and venoocclusive diseases. $^{20}$ Santiago E. Rossi et al have enumerated a detailed description of pathological background of these changes in their study.

Some of the most commonly encountered radiological manifestations are alveolar and interstitial infiltrates, pulmonary haemorrhage and capillary leak syndrome as stated by Jean $M$ Torrisim. $^{15}$ Drugs like bevacizumab have well known association with pulmonary haemorrhage ${ }^{21}$ giving appearance of patches of consolidation.

\section{MATERIALAND METHODS}

This retrospective study was conducted in Kuwait Teaching Hospital. All follow up Chest CT scans had been done on 16 slice Toshiba (specifications) with IV contrast. Patients receiving less than three chemotherapy cycles were not included in the study. Number of total chemotherapy cycles and time lapse since last cycle were documented. The images were reviewed on work station in axial, sagittal and coronal cuts. Images with positive findings were reviewed by three radiologists and were discussed. Chemotherapy related findings were analysed, categorized and tabulated. Incidental pulmonary findings were tabulated separately. All data collection and analysis was done on SPSS version 24.

\section{Definitions}

Chemotherapy: cytotoxic drugs used for cancer treatment.

Post chemotherapy lung toxicity: Side effects of any drug; chemotherapeutic drugs in our case.

CT patterns of post chemotherapy lung toxicity: These were categorized as ground glass haze, ground glass with bronchiectasis, ground glass haze with septal thickening giving crazy paving appearance, patches of consolidation and reticular thickening.

\section{RESULTS}

We reviewed chest CT scans of a total of 204 patients retrospectively. The ages of the patients was grouped in clusters of 10 years. 31 to 40 years age group had the highest frequency of up to $32.8 \%$ of total patients. Male to female ratio was 30.9:69.1 with a total of 63 male and 141 female patients.

Primary malignancy was documented and has been presented in Table No.1. We noticed that the largest number of patients was noticed in lymphoma group comprising of 37 patients forming $18.1 \%$ of total patients. The second largest group was that of breast carcinoma patients comprising of 32 patients making $15.1 \%$ of total patients. This was followed by oesophageal, stomach and intestinal origin carcinomas.

The number of chemotherapy cycles received by individual patients was documented ranging from 3 cycles up to 14 cycles with the mean of 5.95 cycles. Up to $22.1 \%$ patients had received 3 cycles of chemotherapy, $21.1 \%$ of patients had received 4 cycles and about $15.2 \%$ patients had received 5 cycles of chemotherapy. Minimum time lapse between the last cycle was 0 months whereas maximum time lapse was up to 84 months with a mean of 4.8 months and standard deviation of 8.57 on both sides. Highest number of patients presented within one month of chemotherapy which were about 63 patients and formed $31.9 \%$ of total patient strata. 
Table No. 1.

\begin{tabular}{|ccc|}
\hline & Frequency & Percent \\
\hline Lymphoma & 37 & 18.1 \\
\hline Breast & 32 & 15.7 \\
\hline Oesophagus & 11 & 5.4 \\
\hline Stomach & 17 & 8.3 \\
\hline small intestinal & 15 & 7.4 \\
\hline large intestine, rectum & 16 & 7.8 \\
\hline Pancreatic & 6 & 2.9 \\
\hline Ovarian & 17 & 8.3 \\
\hline Endometrial & 6 & 2.9 \\
\hline Pleural & 3 & 1.5 \\
\hline bone or cartilage origin & 6 & 2.9 \\
\hline Others & 38 & 18.6 \\
\hline
\end{tabular}

Table No. 2.

\begin{tabular}{|ccc|}
\hline Chest CT findings & Frequency & Percent \\
\hline $\begin{array}{c}\text { ground glass haze } \\
\text { groung glass haze with } \\
\text { bronchiectasis }\end{array}$ & 10 & 43.5 \\
\hline Consolidations & 4 & 17.4 \\
\hline reticular thickening & 6 & 26.1 \\
\hline ground glass with septal thickening & 1 & 4.3 \\
\hline Total & 2 & 8.7 \\
\hline
\end{tabular}

Table No. 3.

\begin{tabular}{|c|c|c|}
\hline Incidental Findings & Frequency & Percent \\
\hline TB & 13 & 22.4 \\
\hline solitary nodule & 11 & 19.0 \\
\hline Mets & 21 & 36.2 \\
\hline Iymphangitis carcinomatosa & 1 & 1.7 \\
\hline pleural effusion & 12 & 20.7 \\
\hline Total & 58 & 100.0 \\
\hline
\end{tabular}

We analysed chest CT for changes related to chemotherapy and incidental findings

irrelevant to chemotherapy separately. (Table 2 and 3).About 135 patients (66.2\% of total patients) did not show any pulmonary abnormality. 11 patients $(5.4 \%)$ showed chemotherapy related pulmonary findings on CT chest. 46 patients $(22.5 \%)$ showed findings which were incidental and irrelevant to chemotherapy. 12 patients (5.9\%) had both chemotherapy related pulmonary toxicity changes and incidental findings. Thus a total of $11.3 \%$ (23) patients had post chemotherapy pulmonary toxicity. CT manifestations of post chemotherapy lung changes were grouped as ground-glass haze, ground glass haze with bronchiectasis, ground glass haze with septal thickening (crazy paving appearance), patches of consolidation and reticular thickening. 10 patients had sole finding of ground glass haze only $(43.5 \%$ of total patients with post chemotherapy pulmonary changes). 4 patient showed ground glass haze with bronchiectatic changes. This formed $17.4 \%$ of the group. Overall 6 patients showed patches of consolidation forming $26.1 \%$ of the diseased group. Only one patient showed reticular thickening as sole manifestation (4.3\%). 2 patients depicted ground glass haze with septal thickening giving a crazy paving appearance $(8.7 \%)$.

Chest findings irrelevant to chemotherapy were tabulated and analysed separately. Overall 58 patients $(28.4 \%$ of total patients ) showed 
incidental lung findings irrelevant to chemotherapy. As expected the highest presentation was that of active and healed TB (13 patients, $22.4 \%$ ) which is very prevalent in our region.11 patients $(19 \%)$ had solitary nodule whereas 21 patients $(36.2 \%)$ had multiple metastatic deposits.12 patients $(20.7 \%)$ showed pleural effusions. One patient showed perilymphatic nodularity with reticular thickening which was labelled as lymphangitis carcinomatosa. Overall 7 patients $(3.4 \%)$ showed bronchiectasis as sole finding. It was difficult to establish its association with chemotherapy regimen.

Kappa test was applied to establish association between number of chemotherapy cycles and positive CT pulmonary toxicity findings related to chemotherapy only taking $P$ Value less than 0.05 as significant. Incidental CT lung findings irrelevant to chemotherapy were not taken into account. Significant association $(p=0.00)$ was noted.

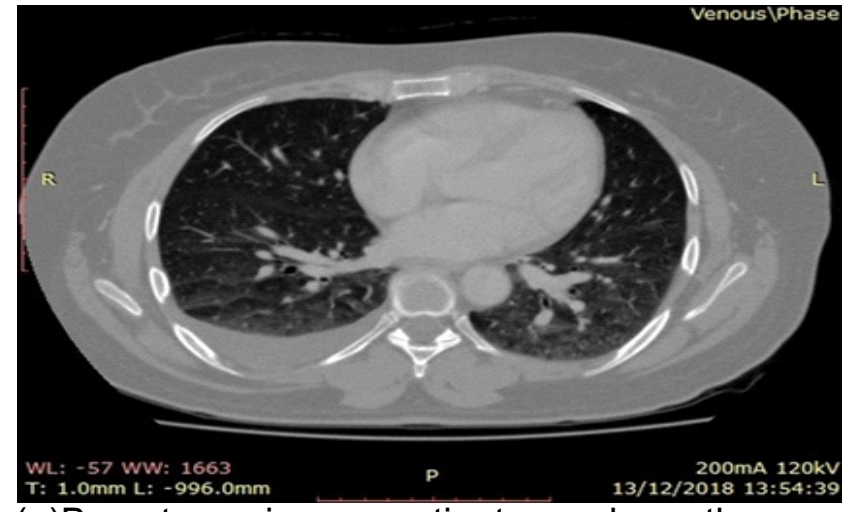

(a)Breast carcinoma patient on chemotherapy. Collapsed consolidation of middle lobe with patent bronchi being evident.Air space shadowing is evident in ligula as well. Minimal right side pleural effusion.

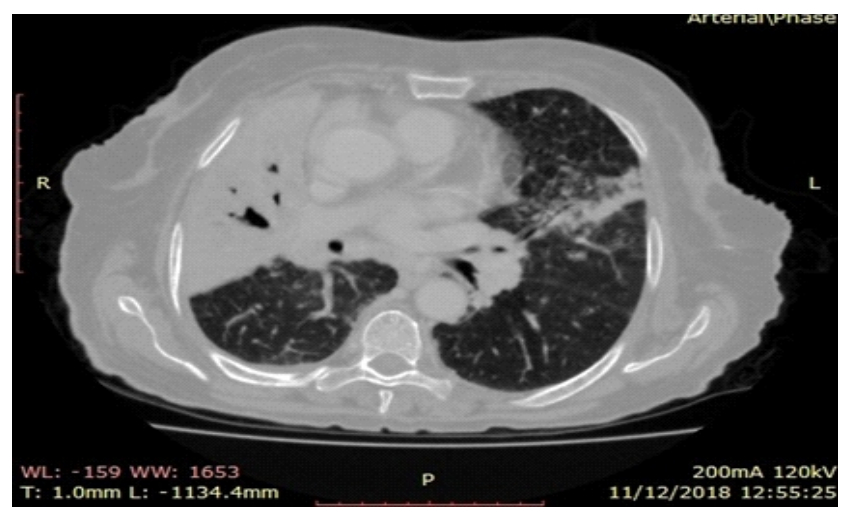

(b) Bilateral pleural effusion as an isolated finding. Minimal ground glass haze in dependant position in keeping with developing pulmonary edema.

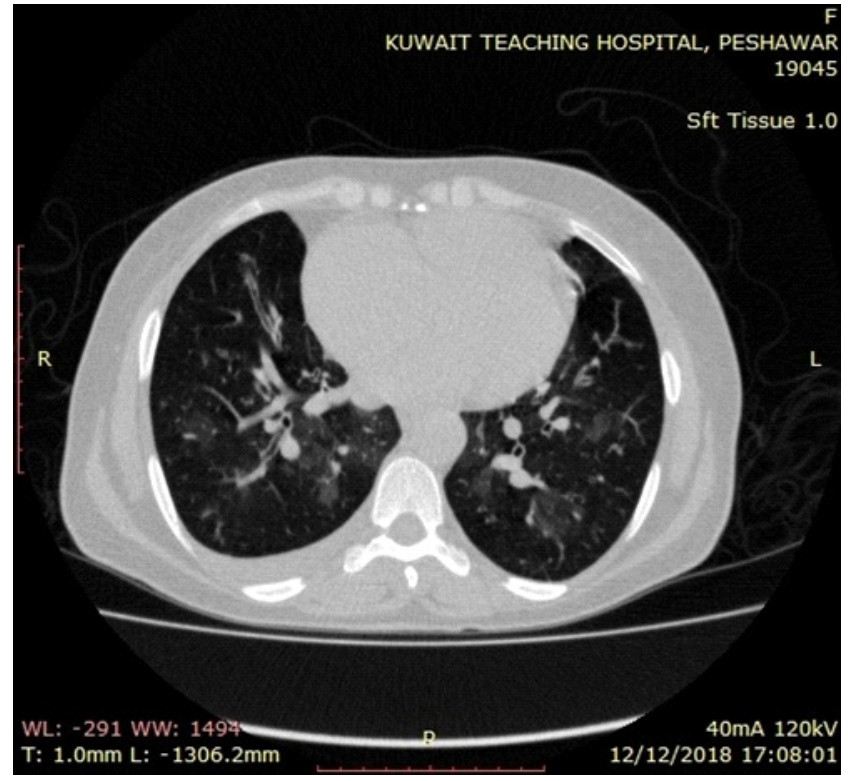

(a)Post chemotherapy Lymphoma patient. Patchy areas of non-specific ground glass haze with minimal right sided pleural effusion. Early bronchiectatic changes in right lung basal segments.

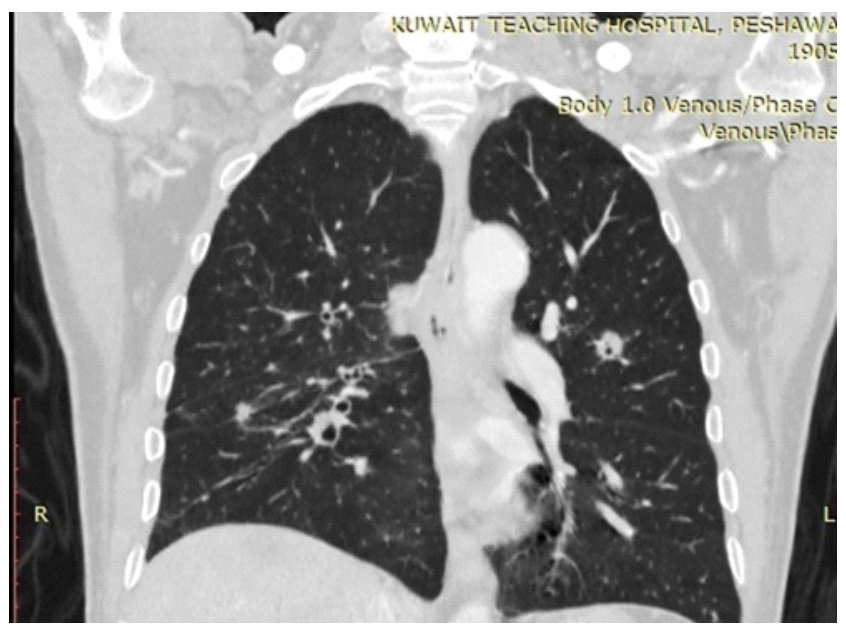

(a)Post chemotherapy patient with a cavitating nodule in left upper lobe which was labelled as fungal cavity .

However no significant association between time lapse since last cycle and post chemotherapy pulmonary toxicity CT findings could be detected $(p=0.16)$. 


\section{DISCUSSION}

Post chemotherapy lung changes are not an infrequent occurring in up to $10 \%$ of patients after receiving chemotherapy. No universally acceptable criteria, for diagnosis of chemotherapeutic drug induced pulmonary toxicity are available. The diagnosis usually depends upon the patient's history of chemotherapy exposure and absence of any other causative agent. Histopathological evaluation is an important adjunct however; tissue diagnosis is not very commonly done just for confirmation of post chemotherapy pulmonary changes. Also another drawback in histopathologic evaluation is that just like radiology no specific criteria for histopathological finding are agreed upon. Thus it is very important on the part of radiologist to document any pulmonary toxicity present and even more to exclude other possibilities like disease progression, metastasis or superadded infection.

In our study we tried to diagnose and classify different CT chest presentations in post chemotherapy patients .MDCT is a very important diagnostic tool partly because of its easy availability, short scan time and partly because it is done for follow up anyways to evaluate chemotherapy response and for re-staging purpose. $^{23}$

In our study we evaluated about 204 cancer patients who had undergone chemotherapy. Patients receiving only up to two cycles of chemotherapy were not included in our study .Out of these 204 patients, 23 showed chemotherapy related pulmonary changes which made about $11.3 \%$ of total reviewed patients. Our results were comparable with a study conducted by $\mathrm{E}$ Helmi et al in 2011 who quoted its incident up to $9 \%{ }^{1}$ and Limper et al who quoted it up to $10 \%{ }^{24}$. Most common finding was that of simple ground glass haze, which was the sole finding in 10 patients (43.5\% of total patients with post chemotherapy pulmonary changes). 4 patients showed bronchiectasis along with ground glass haze which formed $17.4 \%$ of this diseased group. This had a presentation of nonspecific interstitial pneumonitis. These changes were attributed to post chemotherapy fibrotic changes explained as bronchiectasis and early fibrotic changes which were beyond resolution power of MDCT giving ground

glass haze. We did not observe any zonal predominance. This was in contradiction to the findings by Umara et al who have coated an epicobasal predominance ${ }^{.11}$

In one patient reticular thickening was the sole manifestation forming $4.3 \%$ of the diseased group. Crazy paving was present in 2 patients forming $8.7 \%$ of the group. This was in accordance with the findings of Eman Helmey et al who documented their main post-chemotherapy pulmonary findings to be consolidation and ground glass opacities ${ }^{1}$. Cleverly et al have conducted a study on post-chemotherapy pulmonary changes and have documented ground glass haze, consolidation, interlobular septal thickening and centrilobular nodules as their main findings. ${ }^{25}$ Torisi et all has also reported various radiographic patterns of pulmonary toxicity secondary to chemo therapy including ground glass haze, consolidations and septal thickening as main findings. ${ }^{26}$ In our study the most common pulmonary finding was that of patchy or generalized ground glass haze. This is in keeping with findings of Endo et al who have documented ground glass haze as the most common radiological pulmonary presentation in post chemo patients. ${ }^{27}$

We also documented and evaluated various pulmonary findings which are not associated with chemotherapy regimen. Overall 50 patient showed various incidental findings not associated to chemotherapy. Out of these the most prevalent finding was that of active and healed pulmonary tuberculosis, which was present in 13 patients forming $22.4 \%$ of this group which was very predictable as pulmonary tuberculosis is very prevalent in our region. These findings included active TB in the form of patches of consolidation and miliary nodules as well as old healed TB in the form of fibro-cavitatory changes prevalent in the apical segments. Second most common finding was that metastatic pulmonary nodules. 11 patients (forming $19 \%$ of this disease group) had solitary nodules. Multiple pulmonary secondary metastatic deposits were documented in 21 patients forming $36.2 \%$ of the group. Umara et al in their study on HRCT lung findings in post chemotherapy patients have documented granulomatous nodules which arise as a result of macrophages reacting with various chemotherapeutic drugs. However in our study we could not establish the association between pulmonary nodules and chemotherapy regimens. One patient presented with reticular thickening and perilymphatic nodules. This was labelled as lymphangitis carcinomatosa. 
7 patients showed bronchiectasis as sole finding .It was not associated with any ground glass haze or any patches of consultation. These were labelled as incidental finding. We documented the number of chemotherapy cycles being received and the time lapse since the last chemo therapy cycle. In our study the number of chemotherapy cycles received ranged from 3 to 14 cycles with mean of 5.95 cycles. The largest number of patients had received 3 to 4 cycles of chemo therapy. Positive association between the number of chemotherapy cycles and development of for chemotherapy related CT findings was present with $P$ value up to 0.00 . We also documented time lapse since last chemo therapy cycle. Most of the patient had presented with in one month of last chemotherapy cycle. We could not establish any association between post chemotherapy changes and time lapse since last cycle.

A clear gender bias was present in our study with male to female ratio of 31.9: 69.1. We also noted that the highest number of for patients had lymphoma forming $18.1 \%$ of the patients. The second highest group was that of breast carcinoma patients making $15.1 \%$ of total patients. However the kind of malignancy and hence chemotherapy regimen did not appear to have any association with pulmonary toxicity.

\section{CONCLUSION}

Ground glass attenuation was found to be the most profounding feature of post chemotherapy lung CT changes.

\section{REFERENCES}

1. Helmy E, El-Badrawy A, Settein M, Abdelghfar M, EL-Hadaad hand Elkhodary T. Multi-dete ctor Computed Tomography (MDCT) Findings of Chemotherapy-Induced Cardiopulmonary; JCTI, 2016; 3(2): 1-10.

2. G Ramadori, S Cameron . Effects of systemic Chem otherapy on the liver. ANN. HEPATOL; 2010;9(2):133-143

3. Roig J. Pulmonary toxicity caused by cytotoxic drugs. Clin Pulm Med. 2006;13:53-62

4. Galvão FH, Pestana JO, Capelozzi VL.Fatal gemcitabine-induced pulmonary toxicity in metastatic gallbladder adenocarcinoma. Cancer. Chemother Pharmacol. 2010;65:607-610

5. Camus P, Bonniaud P, Fanton A, Camus C, Baudaun N,Foucher P. Drug-induced and iatrogenic infiltrative lung disease. Clin Chest Med 2004; 25:479-519

6. Danson S, Blackhall F, Hulse P, Ranson M. Interstitial lung disease in lung cancer: separating disease progression from treatment effects. Drug Saf 2005; 28: 103-13.
7. Ohno R. Twenty-seven cases of drug-induced interstitial lung disease associated with imatinib mesylate. Leukemia 2006; 20: 1162-4

8. Schwaiblmaer M, Behr W, Haeckel T, Märkl B, Foerg W Berghaus T. Drug Induced Interstitial lung disease. Open Respir Med J. 2012;6: 63-74. doi: 10.2174/1874306401206010063. Epub 2012 Jul 27.

9. Parish J, Muhm J, Leslie K. Upper lobe pulmonary fibrosis associated with high-dose chemothearapy containing $\mathrm{BCNU}$ for bone marrow transplantation. Mayo Clin Proc 2003; 78: 630-4

10. Perran F. Yumuk, Kefeli U ,Ceyhan B ,Faysal Dane ,Basak T,Eroglu, etal. Pulmonary toxicity in patients receiving docetaxel chemotherapy. Med Oncol. Dec;27(4):1381-8. DOI 10.1007/s12032-009-9391-9

11. Umer US, Alam S, Ghuas S, Gul S, Arif QA. HRCT Lung findings in postchemotherapy patientsEmerging challenge for radiologists. JPMI 2015;(4): 670-78

12. Abu Yehia Z, Mikhaeel GN, Smith G Pinnix CC, Milgrom SA, Tang C. etal. Does Bleomycin Lung Toxicity Increase the Risk of Radiation Pneumonitis in Hodgkin Lymphoma? Int J Radiat Oncol Biol Phys. 2016 D e c $1 ; 96$ ( 5 ): $951-958$. doi10.1016/j.jirobp.2016.08.018. Epub 2016 Aug 22

13. Shippee BM, Bates JS, Richards KL. The role of screening and monitoring for bleomycin pulmonary toxicity. J Oncol Pharm Pract. 2016 Apr;22(2):30812. doi: 10.1177/1078155215574294. Epub 2015 Mar 2. Review.PMID: 2573627

14. Kalemci S, Akpınar O, Dere Y, Sarıhan A, Zeybek A, Tanriverdi Ö.Kardiochir Torakochirurgia. Efficacy of clarithromycin as a protective agent in the methotrexate-induced pulmonary fibrosis model. Pol. 2018 Dec;15(4):209-212. doi: 10.5114/kitp.2018.80915. Epub 2018 Dec 31.PMID:30647742

15. Torrisi JM1, Schwartz LH, Gollub MJ, Ginsberg MS, Bosl GJ, Hricak H. CT findings of chemotherapyinduced toxicity: what radiologists need to know about the clinical and radiologic manifestations of chemotherapy toxicity. Radiology. 2011 Jan;258(1):41-56. doi: 10.1148/radiol.1009212

16. Min JH, Lee HY, Lim H, Ahn MJ, Park K, Chung MP , Kyung Lee KS. Drug-induced interstitial lung disease in tyrosine kinase inhibitor therapy for nonsmall cell lung cancer: a review on current insight. Cancer Chemother Pharmacol (2011) 68:10991109.doi 10.1007/s00280-011-1737-2

17. Tereso A, Carreto L, Baptista M, Almeida MA.Interstitial Lung Disease Induced by Crizotinib in Non-Small-Cell Lung Cancer.Acta Med Port. 2019 Mar 29;32(3):236-239. doi: 10.20344/amp.9456. Epub 2019 Mar 29.

18. Miura Y, Saito Y, Minegishi Y, Azuma A, Gemma A. Interstitial lung disease associated by gemcitabine chemotherapy in non-small lung cancer patients: Analysis based on the data in practical use with confirmed denominator .European Respiratory Journal 2011 38: p3741; 
19. Deng SM, Huang JA, Chen YB. Fatal interstitial lung disease associated with AZD9291. J Cancer Res Ther. 2018.Dec;14(Supplement):S1227-S1229. doi: 10.4103/0973-1482.199380

20. Rossi SE, Erasmus JJ, McAdams HP, Sporn TA, Goodman PC. Pulmonary drug toxicity: radiologic and pathologic manifestations. Radiographics. 2000 Sep-Oct;20(5):1245-59.

21. Herbst RS, O'Neill VJ, Fehrenbacher L. Phase II study of efficacy and safety of bevacizumab in combination with chemotherapy or erlotinib compared with chemotherapy alone for treatment of recurrent or refractory non small-cell lung cancer. J Clin Oncol 2007;25(30):4743-4750
22. Johnson DH, Fehrenbacher L, Novotny WF. Randomized phase II trial comparing bevacizumab plus carboplatin and paclitaxel with carboplatin and paclitaxel alone in previously untreated locally advanced or metastatic non-small-cell lung cancer.J Clin Oncol 2004;22(11):2184-2191.

23. Das C, Seith A, Mukhopadhyay S.Thoracic Application of Multi-Detector CT.Indian J Chest Dis Allied Sci. 2007;49:29-36.

24. Limper $\mathrm{AH}$. Chemotherapy-induced lung disease. Clin Chest Med. 2004;25:53-64.

25. Cleverley JR, Screaton NJ, Hiorns MP. Drug-induced lung disease: Highresolution CT and histological findings.Clin Radiol. 2002;57:292-299. 